\title{
Equilibrium shapes and faceting for ionic crystals of body-centered-cubic type
}

\author{
Enrico Carlon \\ INFM, and Dipartimento di Fisica, Università di Padova, I-35131 Padova, Italy
}

Henk van Beijeren

Institute for Theoretical Physics, Utrecht University, P.O. Box 80006, 3508 TA Utrecht, The Netherlands

(Received 5 June 2000)

\begin{abstract}
A mean-field theory is developed for a calculation of the surface free energy of the staggered body-centered solid-on-solid (or six vertex) model as function of the surface orientation and temperature. The model approximately describes surfaces of crystals with nearest neighbor attractions, and next nearest neighbor repulsions. The mean-field free energy is calculated by expressing the model in terms of interacting directed walks on a lattice. The resulting equilibrium shape is very rich with facet boundaries and boundaries between reconstructed and unreconstructed regions, which can be either sharp (first order) or smooth (continuous). In addition, there are tricritical points where a smooth boundary changes into a sharp one, and triple points where three sharp boundaries meet. Finally our numerical results strongly suggest the existence of conical points, at which tangent planes of a finite range of orientations all intersect each other. The thermal evolution of the equilibrium shape in this model shows a strong similarity to that seen experimentally for ionic crystals.
\end{abstract}

PACS number(s): 64.60.Cn

\section{INTRODUCTION}

Crystals in thermal equilibrium are typically composed of flat regions (facets) corresponding to high symmetry directions in the crystal lattice, and possibly some rounded parts. At sufficiently low temperatures the equilibrium crystal shape is dominated by the facets, while with increasing temperature more and more rounded regions occupy larger areas of the crystal surface. At a given temperature a facet may shrink completely and disappear from the equilibrium shape; this corresponds to a roughening transition, which is characterized microscopically by the vanishing of the step free energy on the facet [1].

Another interesting phenomenon occurring on crystal surfaces is that of faceting, or phase separation of unstable orientations $[1,2]$. An orientation is unstable (or metastable) when the total surface free energy of the crystal can be lowered by replacing that orientation by a combination of other orientations, connected to each other under sharp edges, with an average orientation equal to the original one. This process is similar to that of liquid-gas phase separation, where the free energy of the system at a given homogeneous density can be lowered by combining the free energies of a denser liquid phase and a more dilute gas phase [3]. In the case of crystal surfaces the step density is the equivalent of the particle density in the liquid-gas phase separation.

A third phenomenon observed frequently on crystal surfaces is reconstruction, implying that the unit cell of the surface in equilibrium is larger than that obtained by making a section through the bulk crystal structure. The most common cause for this is lowering of surface energy due to the rearrangement of atoms in the surface layer. Raising the temperature often destroys reconstructions at a deconstruction temperature as a result of increasing entropy. In many cases there is a subtle interplay between reconstruction and the other two phenomena.

In this paper we describe the thermal evolution of the equilibrium shape for a model describing equilibrium surfaces of crystals of body-centered-cubic (bcc) type. Using a mean-field approximation, we calculate the surface free energy as function of the surface orientation and temperature. The model has a rich phase diagram showing roughening, faceting, and deconstruction transitions, and it provides an approximate description of ionic crystals of $\mathrm{CsCl}$ type, where ions of the same type repel each other, while there is a strong attraction between nearest neighbors, which are oppositely charged.

The paper is organized as follows: In Sec. I, we introduce the model, and in Sec. II we present the mean-field approximations that we employ to calculate the surface tension of the crystal as a function of surface orientation and temperature. In Sec. III we discuss the evolution of the equilibrium crystal shape as function of the temperature. In Sec. IV we summarize the results obtained, and make a comparison with known models and experiments showing similar features.

A preliminary account of this work was already presented in Ref. [4]. Here we develop a different mean-field theory which has several advantages over that of the previous approach.

\section{MODEL}

We consider a bcc crystal composed of two different types of atoms, say $A$ and $B$, which occupy the sites of the two interpenetrating cubic lattices that form the bcc structure. In the solid-on-solid approximation the surface configurations are given by integers $h_{i}^{A}$ and $h_{j}^{B}$ describing the heights of the surface atoms with respect to a reference plane. $h_{i}^{A}$ and $h_{j}^{B}$ are odd and even integers, respectively.

We consider the Hamiltonian [5]

$$
\begin{aligned}
H= & J_{0} \sum_{\langle i j\rangle}\left(\left|h_{i}^{A}-h_{j}^{B}\right|-1\right)+\frac{\epsilon}{2} \sum_{(k l)}\left|h_{k}^{A}-h_{l}^{A}\right| \\
& +\frac{\epsilon+2 \delta}{2} \sum_{(m n)}\left|h_{m}^{B}-h_{n}^{B}\right|,
\end{aligned}
$$




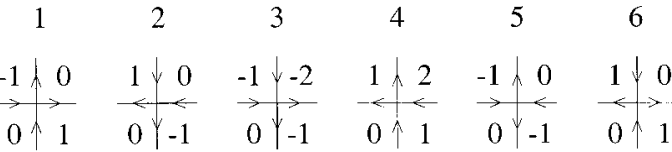

$$
\begin{aligned}
& \begin{array}{lllllll}
\varepsilon & \varepsilon & \varepsilon & \varepsilon+2 \delta & \varepsilon+2 \delta & 0 & 0
\end{array} \\
& \times: \begin{array}{llllll}
\varepsilon+2 \delta & \varepsilon+2 \delta & \varepsilon & \varepsilon & 0 & 0
\end{array}
\end{aligned}
$$

FIG. 1. The six vertices and their energies in the two distinct sublattices $(-$ and $\times$ ). At each vertex, the four integers denote possible height variables.

where the sums are constrained to neighboring $A B, A A$, and $B B$ pairs $(\langle\cdot\rangle$ and $(\cdot)$ denote summations over nearest and next nearest neighbors, respectively).

We take the limit $J_{0} \rightarrow \infty$, so that height differences between neighboring atoms are restricted to their minimal value $( \pm 1)$, and the model can be mapped onto a six vertex model [6,7]. The correspondence between vertices and height variables is shown in Fig. 1. Each vertex satisfies the ice rule, requiring that two of its arrows point inward and two point outward. We stress that if $\delta \neq 0$ the model is mapped onto a staggered six vertex model with the vertex lattice subdivided into two sublattices on which the vertices $1, \ldots, 4$ have different energies, as shown in Fig. 1. As the two representations are completely equivalent in the rest of the paper we will sometimes use the term BC-SOS (bodycentered solid-on-solid) model, and sometimes six vertex model.

We take $\epsilon<0$ and $0<\delta \ll-\epsilon$. This model may give a good approximate description of ionic crystals, where $A$ and $B$ are ions with opposite charges. With our choice of energies neighboring $A$ and $B$ atoms strongly attract each other $\left(J_{0}\right.$ $\rightarrow \infty$ in the model), and atoms of the same type repel each other $(\epsilon, \epsilon+2 \delta<0)$; thus the model may be expected to give at least a good qualitative picture of real ionic crystals, even though further neighbor interactions are ignored. By choosing $\delta>0$ we assumed that in addition to Coulomb repulsion between equal species there is some other contribution to the Hamiltonian, which makes the interaction energies between next nearest neighbor $A A$ and $B B$ pairs slightly different.

The ground state, when $\epsilon$ is negative, describes a $c(2$ $\times 2)$ reconstructed $(001)$ facet with one component $(B)$ at a constant height (say, $h_{j}^{B}=0$ ), and the other $(A)$ with alternat-

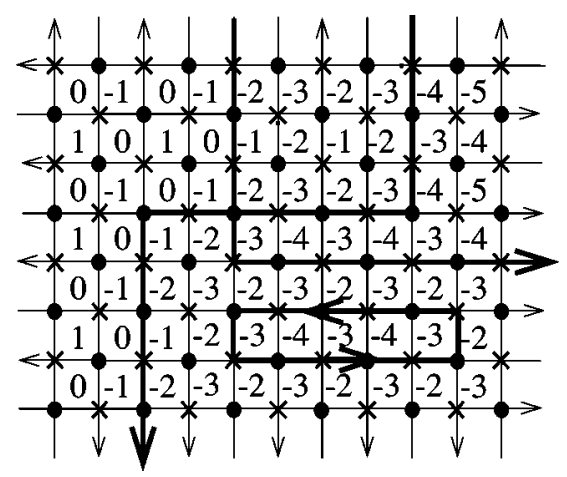

FIG. 2. Connected walks of reversed arrows (thick lines) denote excitations with respect to the Manhattan background (thin lines). Integers are height variables in the BC-SOS representation. ing heights $\left(h_{i}^{A}= \pm 1\right)$ above and below the first one. In the vertex lattice all the arrows point alternatingly up and down or left and right, a configuration which we will refer to as a "Manhattan lattice." Figure 2 shows some elementary excitations of the ground state (thick lines denote excitations with respect to the Manhattan ground state configuration, indicated by thin lines). Reversed arrows on a closed loop produce a closed terrace of surface atoms two lattice units higher (or lower) than their ground state heights. A connected path of reversed arrows running between two boundaries of the Manhattan lattice corresponds to a step on the (001) facet for the BC-SOS model. On the six vertex lattice such a path can be described as a self avoiding walk that cannot visit the same bond twice, but is allowed to cross itself. In reversing arrows the step/walk has to preserve the ice rule, and this limits its possible trajectories. At each noncrossing site, the walk has two options: either to go straight, maintaining its previous direction, with an energy cost of $2 \delta$, or to turn in the direction allowed by the Manhattan lattice, which costs an energy $-\epsilon$ (since we consider the case 0 $<\delta \ll-\epsilon$, typically walks will be composed of very long segments with rare turns). Steps can cross each other, as shown in Fig. 2; step crossings are energetically favored: at a crossing point a ground state vertex is replaced by a vertex with the same energy with all four arrows reversed. There is a gain in energy of $4 \delta$, in comparison to the energy the system would have to pay for two straight noncrossing segments of unit length.

The six vertex model describes not only the (001) surface of a bcc crystal, but also all the side orientations $(t s 1)$ with $|t|+|s| \leqslant 1$. Given a configuration of vertices, the horizontal and vertical polarizations $q$ and $p$ are defined by

$$
q=n_{\uparrow}-n_{\downarrow}, \quad p=n_{\rightarrow}-n_{\leftarrow},
$$

where $n_{\uparrow}, n_{\downarrow}, n_{\rightarrow}, n_{\leftarrow}$ are the densities of up, down, left, and right arrows, respectively. A fully polarized state, namely, a state with all arrows pointing (say) up and to the right describes a (011) facet of the bcc crystal. The relationships between the variables $t, s$ and $p, q$ are given by $t=(p$ $+q) / 2, s=(q-p) / 2$. Please note we have chosen the principal axes of the crystal under angles of $45^{\circ}$ with the principal axes of the vertex lattice.

Figure 3 shows excitations on (011) type facets. In this

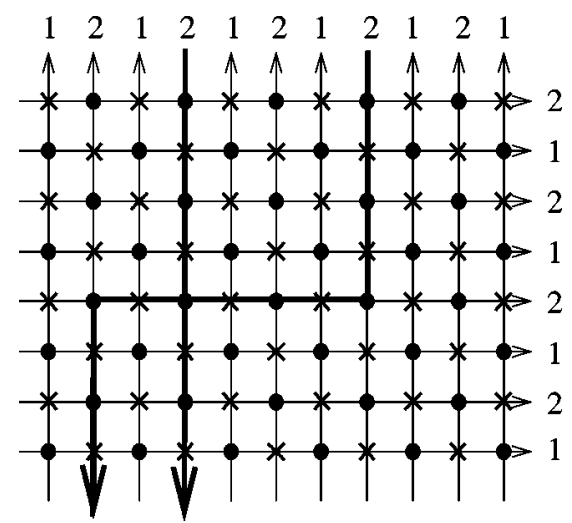

FIG. 3. Excitations (thick lines) with respect to the fully polarized state $p=q=1$, corresponding to steps on the (011) facet. We distinguish between even and odd columns and rows. 
case no closed loops are possible, since they would violate the ice rule, and excitations can be described as directed walks on the lattice, i.e., walks that can only step down or to the left. Two walks cannot visit the same lattice bond, but they can cross each other, as shown in the figure. The meanfield calculation of the free energy of the six vertex model presented in this paper takes as starting point the fully polarized state $q=p=1$. The rows and columns are subdivided into even and odd ones as in Fig. 3; the polarizations $p$ and $q$ are given by

$$
p=\frac{p_{1}+p_{2}}{2} \text { and } \quad q=\frac{q_{1}+q_{2}}{2}
$$

in terms of the sublattice polarizations $p_{1}, p_{2}, q_{1}$, and $q_{2}$. In states with $q=q_{1}=q_{2}$ and $p=p_{1}=p_{2}$ arrows are reversed with respect to the fully polarized state with equal probabilities on the two sublattices, i.e., the surface is unreconstructed. If, on the other hand, one finds states with $q_{1} \neq q_{2}$ or $p_{1} \neq p_{2}$, the surface is reconstructed. Since the energy of a configuration is invariant under the interchange of sublattices 1 and 2 [or, equivalently, a translation over the lattice vector $(1,1)$ on the vertex lattice], each reconstructed state is degenerate with another one with the values of $p_{1}, q_{1}$ and $p_{2}, q_{2}$ interchanged. For either of the states the 1-2 exchange symmetry is spontaneously broken. For example, the two Manhattan states are given by $q_{1}=p_{1}=-q_{2}=-p_{2}= \pm 1$.

\section{CALCULATION OF THE MEAN-FIELD FREE ENERGY}

\section{A. Energy and ground state properties}

It is instructive, before presenting the details of the meanfield approach, to consider the ground state properties of the model, as this will provide already important information on the low temperature behavior of the system. Throughout the rest of the paper we set the ground state energy of the Manhattan state equal to zero by a shift of all the vertex energies over an amount $\epsilon$, so the lowest vertex energy becomes zero. With this convention the energy per site of the fully polarized state (where all arrows point, say, up and to the right) becomes $\delta$. We will take this state as starting point for our mean-field calculations. All other allowed vertex configurations can be represented by a set of directed walks on the lattice, as illustrated in Fig. 3. The lattice points will be distinguished into four different types $i j$, with $i, j=1,2$. The 11 points for example will be the crossing points of odd rows with odd columns of arrows. For a given configuration of directed walks $c_{i j}$ and $t_{i j}$ will indicate the total numbers of crossings and turns, respectively, at sites of type $i j$.

For instance, for the configuration of Fig. 3 one has $c_{22}$ $=1, c_{11}=c_{12}=c_{21}=0$ and $t_{22}=2, t_{11}=t_{12}=t_{21}=0$. An isolated straight path of reversed arrows on the fully polarized state does not change the energy, since it is formed by a collection of vertices with alternating energies $2 \delta$ and $-2 \delta$, which sum to zero. However, crossings and turns do contribute to the energy. A given configuration with $c_{i j}$ crossings and $t_{i j}$ turns at sites of type $i j$ has a total energy

$$
\begin{aligned}
E\left(c_{i j}, t_{i j}\right)= & N^{2} \delta-4 \delta\left(c_{11}+c_{22}\right)+4 \delta\left(c_{12}+c_{21}\right)-\epsilon\left(t_{11}+t_{22}\right) \\
& -(\epsilon+2 \delta)\left(t_{12}+t_{21}\right),
\end{aligned}
$$

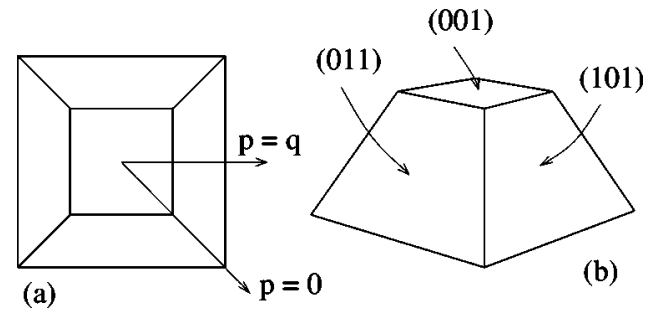

FIG. 4. The equilibrium crystal shape at $T=0$.

since for crossings on 11 or 22 sites one has a gain of energy $4 \delta$, crossings on 12 or 21 sites cost an energy $4 \delta$, while turns on sites of types 11,22 or 12,21 cost energy $-\epsilon$ and $-\epsilon-2 \delta$ respectively.

This expression indeed becomes minimal for the Manhattan states, where one has $c_{11}=c_{22}=N^{2} / 4$ and $c_{12}=c_{21}=t_{i j}$ $=0$. One may look for the minimal energy of states with a homogeneous distribution of polarization on each of the sublattices. For given sublattice polarizations $q_{i}$ and $p_{i}$ the number of crossings may be estimated as

$$
c_{i j}=\frac{N^{2}}{16}\left(1-p_{i}\right)\left(1-q_{j}\right),
$$

an expression that becomes exact if no turns are allowed at all (as occurs at zero temperature). Inserting this value into Eq. (4) and minimizing with respect to $q_{i}$ and $p_{i}$ at fixed 0 $\leqslant q, p \leqslant 1$ one finds the homogeneous ground state energy per vertex,

$$
\epsilon_{0}(p, q)=\delta(p+q)-\delta p q
$$

which is obtained either for $q_{1}=2 q-1, p_{1}=2 p-1, p_{2}$ $=q_{2}=1$ or for $q_{1}=q_{2}=1, p_{2}=2 p-1, q_{2}=2 q-1$. These solutions describe doubly degenerate reconstructed surfaces, with the Manhattan states obtained in the limit $q, p \rightarrow 0$.

However, the homogeneous ground state energy [Eq. (6)] is a nonconvex function of $p$ and $q$. It is well known (see, for instance, Ref. [1]) that instabilities arise when the free energy per unit of projected area is a nonconvex function of the surface orientation. These will give rise to faceting of surfaces with orientations in a nonconvex range. The equilibrium shape constructed from Eq. (6) is shown in Fig. 4; it consists of flat facets only: the "top" facet (001) and "side" facets (011), (101), (0 $\overline{1} 1)$, and ( $\overline{1} 01)$.

Note that Eq. (6) implies that instabilities ought to persist over some range of temperatures, where entropic effects are not sufficiently strong to turn the concave free energy into a convex (i.e., stable) one. The only exception to this will be found at the lines $q=0$ or $p=0$, where the bilinear term in Eq. (4) vanishes. These lines correspond to orientations between the (101) and (011) facets of Fig. 4. It is natural then to expect that finite temperature effects (i.e., the appearance of rounded regions) will first manifest themselves along the common edges of these facets. Summarizing, these simple considerations related to the $T=0$ properties of the system lead us to conclude that (1) sharp edges between the (001) and (011) facets will persist at finite temperatures, and (2) the corners between the facets and the edges between the (011) type facets will probably become rounded first. 


\section{B. Entropy and free energy}

In the sequel we will focus on the behavior of the system for $\delta \ll-\epsilon$, and restrict ourselves to temperatures that are not too high, when directed walks are typically composed of long straight segments, because the Boltzmann weight for a turn $\exp (\beta \epsilon)$, is very small. As a result of this, different walks can hardly develop any local correlations, and the mean-field analysis should be very accurate.

For given sublattice polarizations $p_{i}$ and $q_{i}$ the numbers of crossings $c_{i j}$ in a homogeneous state can still be estimated to be given by Eq. (5), thanks to the absence of correlations between directed walks. The energy of such a state is then given by Eq. (4), with $c_{i j}$ following from Eq. (5). To obtain the free energy at a nonzero temperature, we need both the average numbers of turns $t_{i j}$ and the entropy of the system. In our mean-field approximation both are obtained from the same calculation.

Let us consider as an example the contribution to the entropy resulting from the $t_{11} / 2$ turns from a column 1 to a row 1 . There are $N^{2}\left(1-q_{1}\right) / 8$ inverted arrows on type- 1 columns pointing toward an 11 site, and $c_{11}$ of those are occupied by crossings. There are thus

$$
\Gamma_{11}^{c \rightarrow r}=\frac{N^{2}}{8}\left(1-q_{1}\right)-c_{11}
$$

sites available for the $t_{11} / 2$ turns. The superscript $c \rightarrow r$ indicates that we are considering turns from columns to rows. For turns from rows to columns one finds, analogously,

$$
\Gamma_{11}^{r \rightarrow c}=\frac{N^{2}}{8}\left(1-p_{1}\right)-c_{11}
$$

Both these equations are readily generalized for turns from rows/columns to columns/rows of either type. Neglecting again correlations, one finds that the number of possible ways of making the turns from a column to a row on sites $i j$ is then given by the binomial coefficient of $\Gamma_{i j}^{c \rightarrow r}$ and $t_{i j} / 2$. Collecting the contributions from all possible turns, one finds

$$
S=k_{B} \sum_{i j}\left[\ln \left(\begin{array}{c}
\Gamma_{i j}^{c \rightarrow r} \\
t_{i j} / 2
\end{array}\right)+\ln \left(\begin{array}{c}
\Gamma_{i j}^{r \rightarrow c} \\
t_{i j} / 2
\end{array}\right)\right]
$$

where $k_{B}$ is Boltzmann's constant.

Combining Eqs. (4), (5), and (9), one finds the total free energy $F=E-T S$ as function of the parameters $p_{i}, q_{i}$, and $t_{i j}$. It is convenient to minimize first with respect to the numbers of turns. The equations $\partial F / \partial t_{i j}=0$ for $i, j=1,2$ yield, to lowest order in $e^{\beta \epsilon}$,

$$
t_{i j}=\frac{N^{2}}{4} \sqrt{\left(1-p_{i}^{2}\right)\left(1-q_{j}^{2}\right)} e^{\beta\left(\epsilon+2 \delta \delta_{i j}\right)},
$$

with $\delta_{i j}$ a Kronecker delta. The dependence on the number of turns $t_{i j}$ can thus be eliminated, and to lowest order in $e^{\beta \epsilon}$ the free energy per site becomes

$$
\begin{aligned}
f\left(q_{1}, q_{2}, p_{1}, p_{2}\right)= & \delta\left[1-\frac{1}{4}\left(q_{1}-q_{2}\right)\left(p_{1}-p_{2}\right)\right] \\
& -\frac{1}{8 \beta} \sum_{i j} e^{\beta\left(\epsilon+2 \delta \delta_{i j}\right)} \sqrt{\left(1-p_{i}^{2}\right)\left(1-q_{j}^{2}\right)}
\end{aligned}
$$

It is more convenient to express the sublattice polarizations as

$$
\begin{aligned}
& q_{1}=q-\alpha, \quad p_{1}=p-\gamma, \\
& q_{2}=q+\alpha, \quad p_{2}=p+\gamma,
\end{aligned}
$$

where $p$ and $q$ are the average slopes of the surface, and $\alpha$ and $\gamma$ can be interpreted as order parameters for the reconstructed state; if they are both zero the polarizations $q_{1}, p_{1}$ and $q_{2}, p_{2}$ of the two sublattices are identical and the surface is in an unreconstructed state.

The mean-field free energy can be found by minimization of (11) for fixed $q$ and $p$ :

$$
f_{\mathrm{MF}}(p, q)=\min _{\{\alpha, \gamma\}} f(q-\alpha, q+\alpha, p-\gamma, p+\gamma)
$$

The unreconstructed state corresponding to $\alpha=\gamma=0$ has a free energy given by

$$
f_{\mathrm{MF}}^{(u)}(q, p)=\delta-C(\beta) \sqrt{\left(1-p^{2}\right)\left(1-q^{2}\right)},
$$

with

$$
C(\beta)=\frac{1}{4 \beta} e^{\beta \epsilon}\left(e^{2 \beta \delta}+1\right)
$$

The solution with $\alpha=\gamma=0$ is always a stationary point of the free energy of Eq. (11); however, it is not always a global free energy minimum, as we will see. For arbitrary $p$ and $q$ we implemented the minimization numerically, except along some symmetry lines where it is possible to solve the problem analytically. We will start discussing these special cases first.

\section{Orientations $0 \leqslant p=q \leqslant 1$}

For $q=p$ we take $\alpha=\gamma$ in Eq. (13), and the free energy to be minimized takes the form

$$
\begin{aligned}
f(\alpha)= & \delta\left(1-\alpha^{2}\right)-\frac{1}{4 \beta} e^{\beta \epsilon}\left\{e^{2 \beta \delta}\left[1-\left(q^{2}+\alpha^{2}\right)\right]\right. \\
& \left.+\sqrt{\left[1-(q-\alpha)^{2}\right]\left[1-(q+\alpha)^{2}\right]}\right\}
\end{aligned}
$$

with $q-1 \leqslant \alpha \leqslant q+1$. Setting $\partial f / \partial \alpha=0$, one obtains the following equation:

$2 \alpha\left\{\delta-\frac{e^{\beta(\epsilon+2 \delta)}}{4 \beta}-\frac{e^{\beta \epsilon}}{4 \beta} \frac{1-\left(\alpha^{2}-q^{2}\right)}{\sqrt{\left[1-(\alpha-q)^{2}\right]\left[1-(\alpha+q)^{2}\right]}}\right\}=0$. 
The solution $\alpha=0$ of course corresponds to the unreconstructed state, for which on the symmetry line $q=p$ the free energy takes a simple parabolic shape:

$$
f_{\mathrm{MF}}^{(u)}(q, q)=\delta-C(\beta)+C(\beta) q^{2} .
$$

The other possible solution of Eq. (17) can be found by defining first $\Delta \equiv \alpha^{2}-q^{2}$; squaring Eq. (17), one obtains a quadratic equation for $\Delta$, with a solution

$$
\Delta=1-q \frac{2 \delta-C(\beta)-\widetilde{C}(\beta)}{\sqrt{[\delta-C(\beta)][\delta-\widetilde{C}(\beta)]}},
$$

with $\widetilde{C}(\beta)=e^{\beta \epsilon}\left(e^{2 \beta \delta}-1\right) / 4 \beta$. This equation can also be rewritten in the form

$$
\alpha^{2}=\left(q_{0}-q\right)\left(\frac{1}{q_{0}}-q\right),
$$

with

$$
q_{0}=\sqrt{\frac{\delta-C(\beta)}{\delta-\widetilde{C}(\beta)}} \leqslant 1 .
$$

Note that the reconstructed solution with $\alpha \neq 0$ exists only for a limited range of temperatures and orientations, namely, for $\delta>C(\beta)$ and $|q| \leqslant q_{0}$, where the right hand side of Eq. (20) and the argument of the square root of Eq. (21) are both positive. Note also that as $q \rightarrow q_{0}^{-}$the reconstruction order parameter $\alpha$ vanishes as $\alpha \sim \sqrt{q_{0}-q}$, i.e., with the meanfield exponent $1 / 2$.

Substituting the value of $\Delta$ given by Eq. (19) into Eq. (16), for the reconstructed free energy one finds

$$
\begin{aligned}
f_{\mathrm{MF}}^{(r)}(q, q)= & 2 \sqrt{[\delta-C(\beta)][\delta-\widetilde{C}(\beta)]} q \\
& -[\delta-C(\beta)-\widetilde{C}(\beta)] q^{2} .
\end{aligned}
$$

In the range of parameters where the reconstructed solution exists, this always has a lower free energy than the unreconstructed solution of Eq. (18). At zero temperatures $(\beta \rightarrow$ $+\infty)$ the reconstructed free energy [Eq. (22)] yields the exact ground state energy obtained in Eq. (6). For $q=q_{0}$ the reconstructed [Eq. (22)] and unreconstructed [Eq. (18)] free energies take the same value with equal derivatives.

Figure 5 shows the free energies (solid lines) along the line $p=q$ for different values of the temperature. The dotted lines are the analytical continuations of the unreconstructed free energy in the regions $\left(q<q_{0}\right)$, where this is not the absolute minimum of the free energy [Eq. (11)]. For sufficiently large temperatures [e.g. curve (d) in Fig. 5], the absolute minimum always corresponds to an unreconstructed surface. Note that at low temperatures the reconstructed free energy [Eq. (22)] is concave, as we expected from the analysis of the ground state energy [Eq. (6)].

Stable orientations can be found from the double tangent or Maxwell construction, which is shown as a dashed line in the figure. In Fig. 5(a) the only stable orientations along the $q=p$ line are $q=0$ and $q=1$. In Fig. 5(b) the Maxwell construction connects the orientations $q=0$ with $q=\hat{q}$, where $\hat{q}$

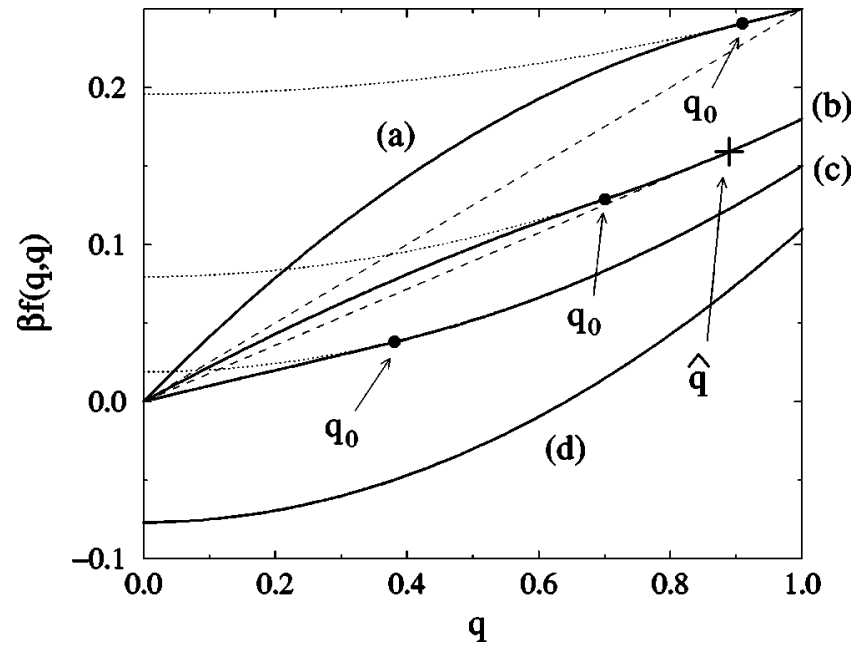

FIG. 5. (Solid lines) mean-field free energies as function of the slope $q$ calculated along the direction $p=q$ and for four different temperatures with $\epsilon=-2.0$ and $\delta=0.2$ : (a) $T=0.80$, (b) $T=1.111$, (c) $T=1.333$, and (d) $T=1.818$. (Dotted lines) analytic continuation of the unreconstructed free energy in the region $q<q_{0}$. (Dashed lines) Maxwell construction connecting stable orientations.

is given in Eq. (36). Therefore, only the orientations in the range $\hat{q} \leqslant q \leqslant 1$ and $q=0$ are stable.

Above a temperature $T_{s}$ obtained from the condition

$$
\delta=C\left(\beta_{s}\right)+\widetilde{C}\left(\beta_{s}\right),
$$

the reconstructed free energy [Eq. (22)] becomes stable [see Fig. 5(c)]. In this case all the surface orientations along the line $q=p$ are stable, and in the range $0 \leqslant q \leqslant q_{0}$ these are reconstructed. The properties of this free energy and of the equilibrium shape along the symmetry axis $p=q$ will be discussed in more detail in Sec. II B 2.

\section{Orientations $p=0,0 \leqslant q \leqslant 1$}

For $p=0$ we minimize the free energy [Eq. (13)] with respect to the parameters $\alpha$ and $\gamma$. The solutions take the form

$$
\begin{gathered}
\alpha^{2}=\left(\tilde{q}_{0}-q\right)\left(\frac{1}{\tilde{q}_{0}}-q\right), \\
\gamma^{2}=\frac{1}{\tilde{q}_{0}} \frac{\tilde{q}_{0}-q}{1-q \tilde{q}_{0}},
\end{gathered}
$$

with

$$
\tilde{q}_{0}=\frac{1}{\delta} \sqrt{\delta^{2}-C^{2}(\beta)}
$$

This yields the following value for the reconstructed free energy,

$$
f_{\mathrm{MF}}^{(r)}(0, q)=\sqrt{\delta^{2}-C^{2}(\beta)} q,
$$

which is valid for $q \leqslant \tilde{q}_{0}$ and $\delta>C(\beta)$.

This free energy is linear as function of the slope parameter $q$. The numerical analysis of the free energy for small 
values of $q$ shows that the reconstructed orientations with small $p$ are not stable, implying that the equilibrium crystal shape has sharp edges along this orientation as we will discuss in detail in Sec. III.

\section{Orientations $q=1,0 \leqslant p \leqslant 1$}

The exact value of the free energy at $q=1$ can be calculated easily. We show here that in this limiting case the mean-field free energy reproduces the exact result. Let us consider a vertex lattice of size $N \times N: q=1$ describes a set of orientations where all the vertical arrows are up, in a state of maximal polarization (the state is composed of vertices 1 and 4). The horizontal arrows can point either to the left or to the right, but once a horizontal arrow at a boundary is fixed, all the arrows connected to it along a horizontal line point in the same direction; otherwise the ice rule would be violated. The average energy per site is $\delta$ since along horizontal lines, there is an alternating sequence of vertices of energies 0 and $2 \delta$. The total entropy of the $N \times N$ lattice is of order $N$, since the vertical arrows are frozen and the horizontal ones along a row are identical. Therefore, the free energy per site becomes

$$
f(p, q=1)=\delta+O(1 / N),
$$

and in the thermodynamic limit $N \rightarrow \infty$ the entropic term can be neglected. This limiting value of the free energy is reproduced correctly by our mean-field calculations at all temperatures. The free energy for vicinal orientations very close to (011) type facets can also be calculated exactly. It just involves calculating the free energy of isolated steps on these facets. This calculation is worked out in the Appendix. It turns out that our mean-field approximation also describes the free energy of these vicinal orientations correctly. This is no surprise, since for these orientations crossings are very rare and unimportant, so that our method for estimating the entropy and the numbers of turns becomes exact.

\section{Orientation $p=q=0$}

The other limiting case to be considered is the orientation with $q=p=0$. In this case, for $\delta>C(\beta)$, Eq. (13) yields two minima with $\alpha=\gamma= \pm 1$, which correspond to the two reconstructed Manhattan states with $f_{\mathrm{MF}}^{(r)}(p=0, q=0)=0$. Since the ground state energy of the system equals zero we expect that in reality $f(p=0, q=0)<0$ at finite temperatures. The reason we do not find this in our mean-field approximation is due to the fact that we have neglected correlations between directed walks. For example, at very low temperatures the most important excitations of the ground state are closed loops around an elementary square on the Manhattan lattice. These obviously exhibit strong correlations; four turns are located on the corners of the same elementary square. However, their contribution to $f$ is of the order $\exp (4 \beta \epsilon)$ and therefore ignoring these terms is consistent within our approximation scheme. Above the temperature $T_{\mathrm{MF}}=1 /\left(k_{B} \beta_{\mathrm{MF}}\right)$, satisfying

$$
\delta=C\left(\beta_{\mathrm{MF}}\right),
$$

the minimum of Eq. (11) corresponds to $\alpha=\gamma=0$, and the orientation $p=q=0$ is unreconstructed, with free energy given by Eq. (14), which is negative as expected. So for this orientation our mean-field theory predicts a phase transition where the reconstruction order parameters ( $\alpha$ and $\gamma$ ) jump from \pm 1 to zero and the step free energy vanishes with a mean-field exponent $1 / 2$, as can be seen from the free energies [Eqs. (22) and (27)].

Transfer matrix calculations [8] strongly suggest that in reality the reconstruction transition and the roughening transition occur at separate temperatures, and that both are continuous. However, the temperature difference between the two transitions decreases very rapidly with increasing $|\epsilon|$ (our estimates [8] yield an approach as $e^{12 \beta \epsilon}$ ), and, as $|\epsilon|$ becomes larger, the increase of $\beta f$ from practically zero to appreciable values occurs within an increasingly narrow range of $\beta \epsilon$. Since we expect our mean-field approximation to become exact in the limit $|\epsilon| / \delta \rightarrow \infty$, the predicted coincidence of roughening and deconstruction, as well as the first order character of this phase transition, are in fact to be expected.

\section{Properties of the free energy for arbitrary $p$ and $q$}

At the points $p=q=q_{0}$ and $p=0, q=\tilde{q}_{0}$ the reconstruction parameter $\alpha$ becomes zero, as follows from Eqs. (20) and (24). One can find the locus $\alpha=0$ for general $p$ and $q$ by setting $q_{1}=q-\alpha, q_{2}=q+\alpha$ and $p_{1}=p-\gamma, p_{2}=p+\gamma$, and requiring that the matrix of the second derivatives of the free energy with respect to the parameters $\alpha$ and $\gamma$ has zero determinant for $\alpha=\gamma=0$. This requirement yields the following condition:

$$
C(\beta)-p q \widetilde{C}(\beta)=\delta \sqrt{\left(1-p^{2}\right)\left(1-q^{2}\right)} .
$$

This line may be interpreted as a spinodal line for the reconstruction transition. Its stable part describes a smooth boundary between reconstructed and unreconstructed regions. As we will see later, part of this line is actually thermodynamically unstable.

At this point we should also discuss the symmetry properties of the surface and its free energy. Obviously the latter should be invariant under the transformations $p \rightarrow-p, q \rightarrow$ $-q$, and $p, q \rightarrow q, p$. Our expression for the unreconstructed free energy satisfies all these requirements. The one for the reconstructed free energy is invariant under the last transformation and under $p, q \rightarrow-p,-q$, but it is not invariant under reflection of $p$ or $q$ alone. A little thinking reveals that we have broken this symmetry by our identification of positive $p$ and $q$ with given arrow directions; if one identifies the opposite horizontal arrow direction with positive $p$ and changes the sign of the actual horizontal polarization, obviously our expression for the free energy does not change. So, to restore the required symmetry, one has to adopt the convention that positive $p$ and $q$ should correspond to the direction of the majority of the horizontal respectively vertical arrows. This does not look unreasonable: our mean-field assumptions obviously work better the smaller the number of overturned arrows with respect to the fully polarized state. On the other hand, in stable reconstructed states the fraction of overturned arrows often is close to one half, so the mean-field description used here may be less accurate than one might like. 


\section{EQUILIBRIUM CRYSTAL SHAPES}

\section{A. Maxwell construction}

Unstable or metastable regions on a free energy surface can be stabilized by applying a Maxwell construction. This amounts to connecting all pairs of points on the free energy surface by tie lines, constructing the lower envelope of the free energy surface combined with the set of all the tie lines, putting this in place of the original free energy surface, and repeating this procedure until it converges. The new points that are generated this way represent "coexisting states" of two or three stable orientations. In other words, the most favorable way for the crystal to realize surfaces of the corresponding orientations is by faceting; that is, by replacing these surfaces with a combination of two or three different surface elements with the same average orientation. Surface orientations that give rise to faceting do not appear in the equilibrium shape of the crystal; they are not thermodynamically stable.

\section{B. Orientations $0 \leqslant p=q \leqslant 1$}

The dashed lines in Fig. 5 show an example of the Maxwell construction applied to the orientations $0 \leqslant p=q \leqslant 1$. From the stable free energy $f$, one finds the equilibrium shape using [1]

$$
x=\frac{\partial f}{\partial p}, \quad y=\frac{\partial f}{\partial q} \quad \text { and } \quad z=f-p \frac{\partial f}{\partial p}-q \frac{\partial f}{\partial q}
$$

up to an arbitrary prefactor. These equations can be used to express $z$ as a function of $x$ and $y$. For the unreconstructed rounded parts of the surface, one has to substitute Eq. (14) for $f$. This yields a shape equation of the form

$$
z=\delta-C \sqrt{\left(1+\frac{x^{2}}{C^{2}}\right)\left(1+\frac{y^{2}}{C^{2}}\right)},
$$

where $C$ was defined in Eq. (15). From Eqs. (31) applied to the unreconstructed free energy, one also finds that $x y$ $=C^{2} p q$; it then follows that the boundary of the $p=q=1$ facet is simply given by a hyperbola:

$$
x y=C^{2} .
$$

Applying the Maxwell construction is not very practical, since the surface free energy depends on two slope parameters. Instead one may obtain the equilibrium shape of the crystal by just applying Eqs. (31) and discarding unstable wings, as described in Ref. [1]. That is, all points with $z$ $>0$ are unstable, as they would require some nonconvex surface part to reach them from the top facet, which is located at $z=0$. Unstable wings can also occur in the region $z \leqslant 0$, as will be shown in the numerical analysis of the equilibrium shape for generic $p$ and $q$.

Along the symmetry line $p=q$ one can use the analytic expressions (22) and (18) for the reconstructed and unreconstructed free energies. One finds that at low temperatures Eq. (31) gives $z>0$, except for the two facets which are then connected directly under a sharp edge as at $T=0$. The shape along the symmetry line is then given by

$$
z=\left\{\begin{array}{lll}
0 & \text { for } & |x|=|y| \leqslant \delta / 2 \\
\delta-2|x| & \text { for } & |x|=|y| \geqslant \delta / 2
\end{array}\right.
$$

At higher temperatures in the vicinity of the $q=p=1$ facet, some unreconstructed orientations become stable. The condition for this to happen is that the Maxwell construction connects $q=p=0$ with $q=p=\hat{q}<1$ [see Fig. 5(b) for an example] or, equivalently, that in Eq. (32) a range of orientations will give rise to $z<0$. Applying Eqs. (31) and (14) for $p, q \rightarrow 1$, one finds that $z=\delta-2 C(\beta)$. Equating this to zero, one obtains the so-called edge rounding temperature $T_{\mathrm{ER}}$ from

$$
\delta=2 C\left(\beta_{\mathrm{ER}}\right)
$$

For $T>T_{\mathrm{ER}}$ there is a range of stable unreconstructed orientations connected to the (001) facet under a sharp edge, where the state $q=p=0$ coexists with a state $q=p=\hat{q}$. The value of $\hat{q}$ can be found either by applying the Maxwell construction or again from the requirement $z(x=y)=0$ in Eq. (32). One finds

$$
\hat{q}=\sqrt{\frac{\delta-C}{C}}
$$

For $T_{\mathrm{ER}}<T<T_{\mathrm{s}}$, with $T_{\mathrm{s}}$ given by Eq. (23), the shape profile is given by

$$
z=\left\{\begin{array}{lll}
0 & \text { for } & |x|=|y| \leqslant \hat{x} \\
\delta-C-x^{2} / C & \text { for } & \hat{x} \leqslant|x|=|y| \leqslant C \\
\delta-2|x| & \text { for } & |x|=|y| \geqslant C,
\end{array}\right.
$$

with $\hat{x}=C \hat{q}$. Note that $z(x)$ has a jump in the first derivative at $x=\hat{x}$ (sharp edge), and in the second derivative for $x=C$ (smooth edge).

As pointed out in Sec. II, above a temperature $T_{\mathrm{s}}$ [see Eq. (23)] there is a range of temperatures where the reconstructed free energy [Eq. (22)] becomes stable. Applying Eqs. (31) to the reconstructed free energy, one finds the shape profile

$$
z=\left\{\begin{array}{lll}
0 & \text { for } & |x|=|y| \leqslant x_{f} \\
A\left(x_{f}-x\right)^{2} & \text { for } & x_{f} \leqslant|x|=|y| \leqslant x_{0} \\
\delta-C-x^{2} / C & \text { for } & x_{0} \leqslant|x|=|y| \leqslant C \\
\delta-2|x| & \text { for } & |x|=|y| \geqslant C,
\end{array}\right.
$$

with

$$
\begin{gathered}
x_{f}=\sqrt{(\delta-C)(\delta-\widetilde{C})}, \\
x_{0}=C \sqrt{\frac{\delta-C}{\delta-\widetilde{C}}} \\
A=-\frac{1}{C+\widetilde{C}-\delta} .
\end{gathered}
$$



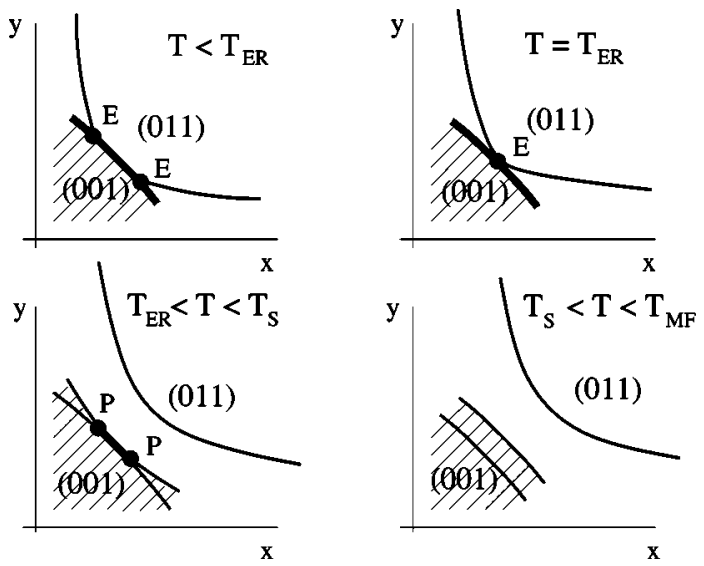

FIG. 6. Thermal evolution of the crystal shape (top view) in the vicinity of the line $x=y$. Thick lines denote sharp edges in the shape. For a detailed description, see the text. Above the temperature $T_{\mathrm{MF}}$ the (001) facet, as well as the reconstructed orientations, disappear from the crystal, and the global shape is described by Eq. (32).

The rounded regions in the range $x_{f} \leqslant|x|=|y| \leqslant x_{0}$ are thus reconstructed and connected smoothly to the top facet (which is also reconstructed), and to the unreconstructed rounded regions. As remarked previously, by approaching the boundary between reconstructed and unreconstructed regions from the reconstructed side, i.e., for $x=y \rightarrow x_{0}^{-}$, one finds that the reconstruction order parameter $\alpha$ vanishes continuously with a mean-field exponent $1 / 2$, as is easily seen from Eq. (20) combined with $x=C q$. At this point the shape profile is also singular, as the second derivative of $z(x)$ has a jump at $x=x_{0}$. Note that the shape is parabolic in the vicinity of the (001) and (011) facets, i.e., $z-z^{\prime} \sim\left(x-x^{\prime}\right)^{2}$. This is typically a mean-field result; the actual exponent ought to be $3 / 2$ instead of 2 , as found in exactly solved models [1]. Finally, at the temperature $T_{\mathrm{MF}}$ [see Eq. (29)], one has $x_{f}, x_{0}$ $\rightarrow 0$, confirming the simultaneous occurrence of a roughening and a deconstruction transition discussed already in Sec. II. For $T \geqslant T_{\mathrm{MF}}$, the rounded part of the crystal is described entirely by Eq. (32).

Figure 6 schematically shows the top view of the equilibrium crystal shape in the vicinity of the line $x=y$ at different temperatures. At low temperatures $T<T_{\mathrm{ER}}$, the (001) and (011) facets are connected to each other along the segment $E E$. The (011) facet boundary, starting from the points $E$, is described by the two branches of the hyperbola given in Eq. (33). The coordinates of points $E$ can be found exactly from the intersection of Eq. (33) with the constant height contour [Eq. (32)] for $z=0$, where the hyperbola intersects the (001) facet. One finds

$$
\begin{aligned}
& x_{E}=\sqrt{\frac{\delta^{2}}{2}-C^{2}+\delta \sqrt{\frac{\delta^{2}}{4}-C^{2}}}, \\
& y_{E}=\sqrt{\frac{\delta^{2}}{2}-C^{2}-\delta \sqrt{\frac{\delta^{2}}{4}-C^{2}}} .
\end{aligned}
$$

The two points of coordinates $\left(x_{E}, y_{E}\right)$ and $\left(y_{E}, x_{E}\right)$ merge into a single one at the edge rounding temperature $(T$

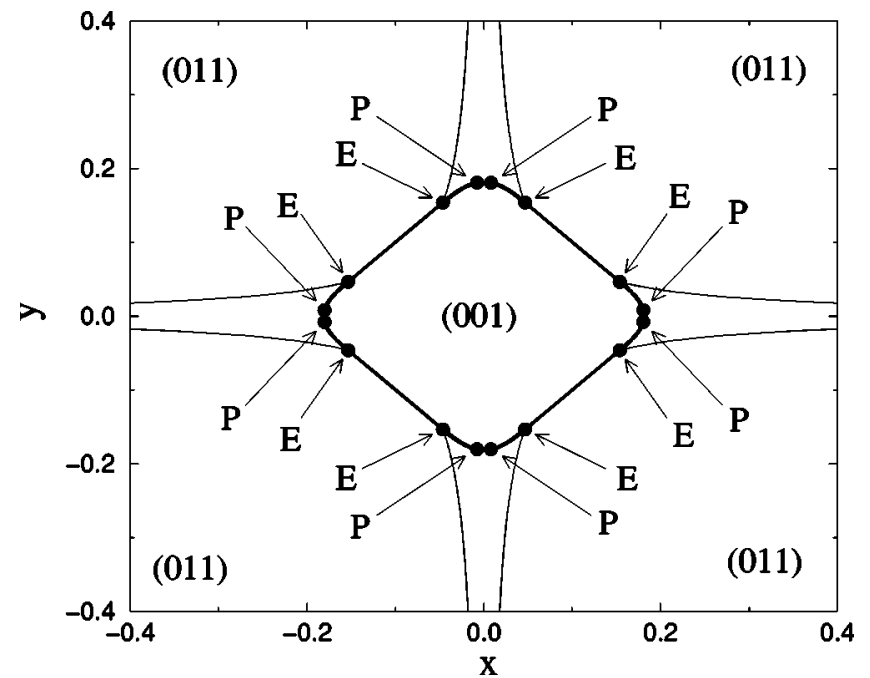

FIG. 7. Equilibrium crystal shape (top view) for $\epsilon=-2, \delta$ $=0.2$ and $T=1.0$, i.e., below the edge rounding temperature $\left(T_{\mathrm{ER}}\right.$ $\approx 1.065$ ). Thick and thin lines denote sharp and smooth facet edges, respectively. The boundaries of the (011) facets with the rounded regions are always smooth. For a detailed description of points $E$ and $P$, see the text.

$=T_{\mathrm{ER}}$ ), where $x_{E}=y_{E}=\delta / 2$. For $T<T_{\mathrm{ER}}$ the boundary between the two points $E$ is described by the equation $x+y$ $=\delta$, as for $T=0$. For $T_{\mathrm{ER}}<T<T_{s}$ the (001) and (011) facets are completely separated from each other. The full analysis for generic $p$ and $q$ shows (more details below) that the (001) facet edge is sharp only between the two points $P$ shown in Fig. 6(c). Beyond these points one finds rounded reconstructed regions and a smooth (001) facet edge. Finally for $T_{s}<T<T_{\mathrm{MF}}$ the (001) facet edge is surrounded by curved reconstructed orientations, and it connects smoothly to the rounded regions along its full circumference in the whole crystal.

\section{General shape}

For generic $p$ and $q$ we have calculated the mean-field equilibrium shape numerically, except outside the boundary given by Eq. (30), where the unreconstructed state prevails, and the equilibrium shape is given by Eq. (32). Figure 7 shows a top view of the equilibrium crystal shape (ECS) for $\epsilon=-2$, and $\delta=0.2$, and for a temperature $T=1.0$, i.e. below the edge rounding temperature $T_{E R} \approx 1.065$. Thick lines mark those parts of the (001) facet boundary which are sharp. The $E E$ segments are sharp and straight as at $T=0$. The $P E$ parts are also sharp; here the (001) facet is connected to unreconstructed rounded regions. The $P P$ parts are smooth, and the (001) facet is connected to reconstructed rounded regions. The shape of the crystal around the points $P$, in the vicinity of the axes $x=0$ and $y=0$, will be discussed later. The boundaries of the (011) facets with rounded regions are always smooth and marked by thin lines originating from points $E$.

Figure 8 shows the ECS for $T=1.25$, i.e. above the edge rounding temperature. Now all facets are separated from each other by rounded regions. The (001) facet boundary is sharp in the part marked by thick solid lines; here the (001) facet is connected to unreconstructed rounded regions. Fig- 


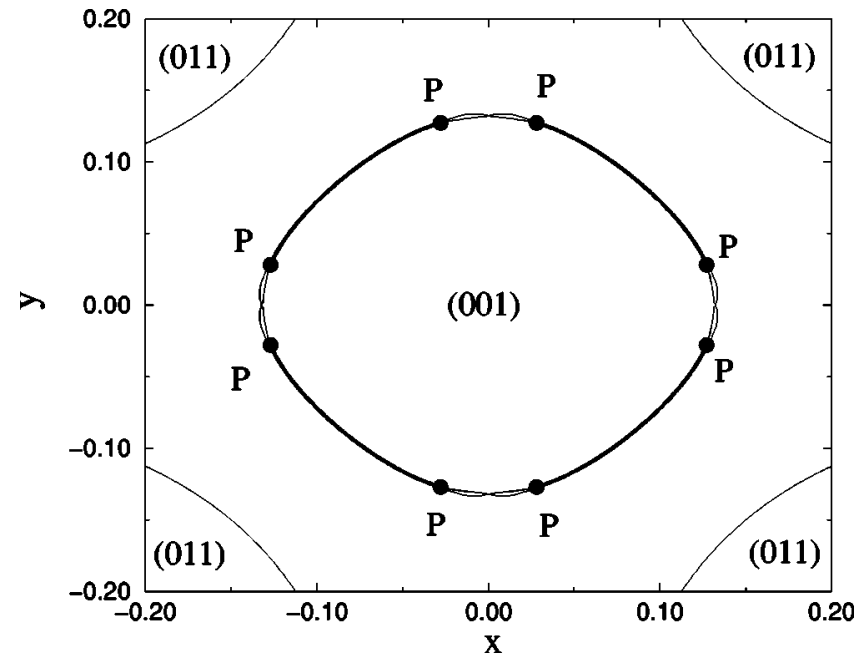

FIG. 8. As in Fig. 7 for a temperature $T=1.25$, i.e., above the edge rounding temperature. The part of the facet edge between the two points $P$, indicated with a thick solid line, is sharp. Between two points $P$, and close to the axes $x=0$ and $y=0$, there are two wings of reconstructed rounded regions, which are shown in some detail for $T=1.111 \ldots$ in Fig. 9.

ure 9 shows a blowup of the equilibrium crystal shape in the vicinity of the $x=0$ axis for $T=1.111 \ldots$ The area below the $P-A-P$ curve belongs to the (001) facet. As in Figs. 7 and 8 , thick solid lines denote sharp edges. Thinner lines are smooth boundaries, either facet edges (as the segments $A-P$ ) or boundaries between the reconstructed and unreconstructed regions (curve $P-C_{\text {trc }}$ ). To facilitate the description of the crystal shape, we have plotted some contour lines corresponding to constant values of $z$. They have been drawn solid in the unreconstructed part of the crystal and dashed in the reconstructed regions. The $(001)$ facet has $z=0$, and the contours correspond to the respective values $z=-0.0001$, $-0.0003,-0.0007,-0.00125$, and -0.0018 . Starting from

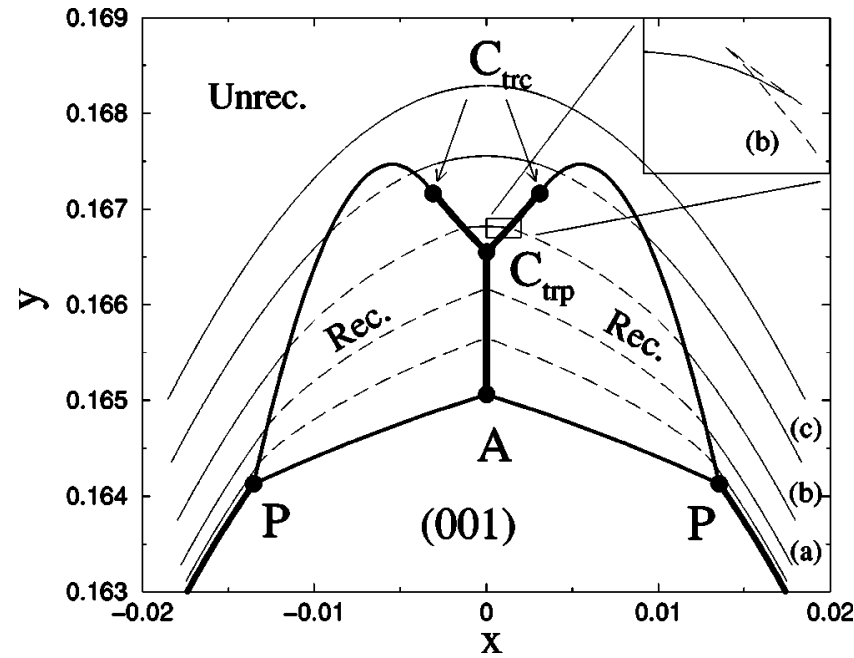

FIG. 9. Top view of an enlargement of the ECS close to the intersection of the edge of the (001) facet with the axis $x=0$, for $\epsilon=-2, \delta=0.2$, and $T=1.111 \ldots$ The thinnest lines denote contours for fixed values of $z$. The (001) facet is located at $z=0$, and the other lines correspond to $z=-0.0001$ and -0.0003 (a), $z=$ -0.0007 (b), $z=-0.00125$ (c), and $z=-0.0018$. the contour line (a) at $x \approx 0.15$, i.e., in the unreconstructed rounded region and shifting toward smaller values of $x$, one first encounters the $P-C_{\text {trc }}$ boundary which separates the unreconstructed region from the reconstructed region; the crystal shape at this boundary is smooth, in the sense that the first derivatives of $z(x, y)$ as functions of $x$ and $y$ are continuous, but higher derivatives are not. Proceeding further along the contour line (a), one terminates in the $A-C_{\text {trp }}$ segment, which is a sharp ridge in the rounded part of the crystal [9]. For the contour line (b), which corresponds to a lower value of $z$, a similar behavior to that for (a) is found, except that the reconstructed region terminates in the segment $\mathrm{C}_{\text {trp }}-\mathrm{C}_{\text {trc }}$ and not at $x=0$. The inset of Fig. 9 shows an enlargement of the contour line (b) in the area around $C_{\mathrm{trp}}-C_{\mathrm{trc}}$. The contour line is obtained from the Legendre transform of the full free energy, without applying the Maxwell construction. This procedure produces an unstable wing which must be eliminated from the shape. Unstable wings are rather easy to identify, and indicate the existence of a sharp boundary in the crystal shape. The contour (c) crosses the boundary in two points, and in both points the boundary is smooth; in this case we did not observe any unstable wing along the line. Point $C_{\text {trc }}$ marks the end of the first order line, and is a tricritical point. Point $C_{\text {trp }}$ where the three first order lines merge is a triple point. Note that the reconstructed region is rather small; this area is hardly visible on the scales of Figs. 7 and 8. As the temperature is increased the reconstructed areas occupy a larger portion of the crystal surface.

The coordinates of point $P$ can be found analytically from the intersection of Eq. (30) with the unreconstructed crystal shape Eq. (32) with $z=0$. One obtains

$$
x_{P}=\sqrt{\frac{\delta^{2}-C^{2}-\widetilde{C}^{2}-\sqrt{\left[\delta^{2}-(C+\widetilde{C})^{2}\right]\left[\delta^{2}-(C-\widetilde{C})^{2}\right]}}{2}},
$$

$$
y_{P}=\sqrt{\frac{\delta^{2}-C^{2}-\widetilde{C}^{2}+\sqrt{\left[\delta^{2}-(C+\widetilde{C})^{2}\right]\left[\delta^{2}-(C-\widetilde{C})^{2}\right]}}{2}} .
$$

At a temperature $T_{s}$ obtained from the condition (23) the two points $P$ merge into a single point $\left(x_{P}=y_{P}\right)$. Above $T_{s}$ the points $P$ disappear, and the whole edge of the (001) facet connects smoothly to the rounded regions surrounding the (001) facet. Still, in the vicinity of the axes $x=y=0$, one has tricritical and triple points as in Fig. 9. As discussed above, there is a temperature $T_{\mathrm{MF}}$ where the (001) facet vanishes (roughening) and the reconstruction simultaneously disappears.

We conclude the discussion of the crystal shape with an analysis of the properties of point $P$, which turn out to be very interesting. A numerical solution of Eqs. (13) and (31) leads to the results illustrated in Fig. 10. Several lines connecting points with equal values of $p / q$ intersect each other at the common point $P$, with a wide range of values for $p$ and $q$ assumed in this point. This corresponds to the geometry of the tip of a cone, where one also has tangent planes of a wide range of orientations. Therefore we will refer to $P$ as as a conical point. An analogous point was found in a nonstag- 


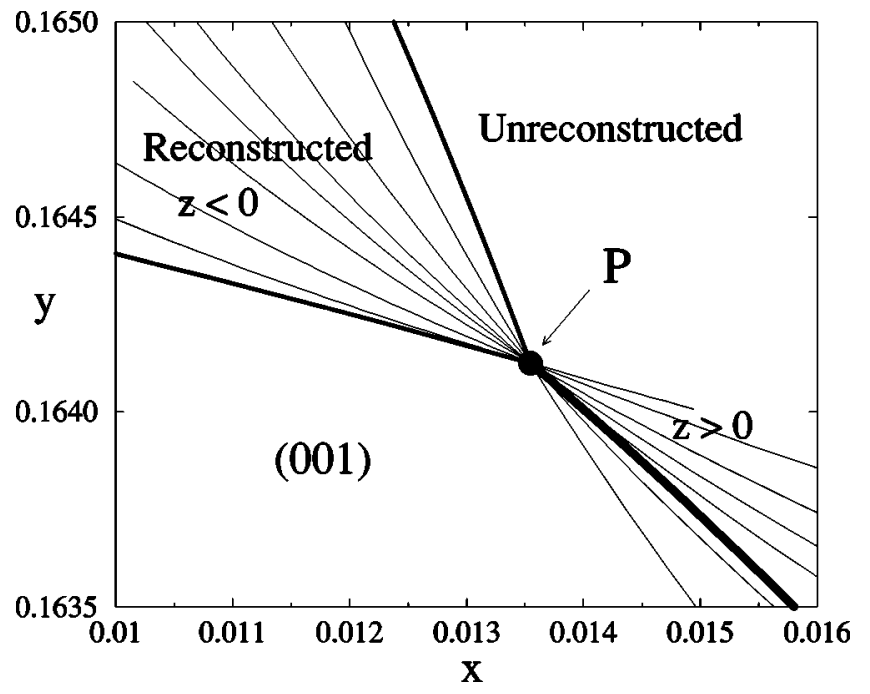

FIG. 10. Enlargement of the top view of the ECS around the point $\mathrm{P}(T=1.111 \ldots)$. Thin lines are trajectories obtained at constant ratios $p / q$. The fact that all these intersect at $P$ indicates that $P$ is a conical point.

gered version of the six vertex model [10], which is exactly solvable. Figure 10 shows an enlargement of the region around point $P$. The temperature is the same as in Fig. 9. The thick solid line denotes the sharp (first order) boundary which opens up in $P$ into two continuous boundaries. The lower one is the smooth (001) facet edge, and the upper one is the boundary between reconstructed and unreconstructed rounded regions. The thin lines are trajectories obtained by keeping the ratio $q / p$ constant; they are indeed seen to intersect each other at $P$. Note that $P$ is at the facet edge; therefore, $z=0$. The parts of the lines to the left of $P$ correspond to $z<0$, and are stable. The parts to the right of $P$ have positive $z$, and describe an unstable wing, which has to be eliminated from the equilibrium shape. In some sense point $P$ is highly degenerate, since a continuous set of values of $q$ and $p$ coexist. In a plot of the free energy vs orientation, i.e., of $f$ as a function of $p$ and $q$, this is reflected in the form of a flat area for a certain range of orientations, namely, those physically realizable orientations that are tangent at the conical point and all their convex combinations. The existence of such a flat area is a direct consequence of Eq. (31); for different points on the $f(p, q)$ surface to give rise to the same $x$, $y$, and $z$, they have to lie in a common plane. From a physical point of view the existence of such a flat area in the $f(p, q)$ surface looks extremely surprising. It corresponds to a finite range of step densities for which the step dependent part of the free energy is strictly proportional to the step density, i.e., steps are apparently noninteracting. It should be stressed, however, that so far almost all our evidence for the conical character of $P$ is numerical. Although the accuracy of our calculations is very high and all our results indicate a conical behavior, we cannot exclude the possibility that on an even finer scale the lines of various $p / q$ will not intersect exactly, and in fact the single conical point $P$ will extend into some folds and creases, the extensions of which certainly have to be very small. However, there is one important piece of analytical support for a strictly conical behavior of $P$, which is the behavior of $f(p, q)$ at the temperature $T_{s}$. Here the two points $P$, as drawn in Fig. 7 , merge into a single point. Ac- cording to Eq. (22) all orientations $p=q$ with $0<q<q_{0}$ $=\sqrt{\widetilde{C}\left(\beta_{s}\right) / C\left(\beta_{s}\right)}$ indeed join at point $P$. This is a limiting case of a conical point, where the opening angle of the cone becomes zero. There seems as little physical reason for the coincidence of all these orientations in $P$ as in the general case, but of course this does not provide a proof for conical behavior in general.

Note that in a previous mean-field calculation [4] the two facets (101) and (011) were found to be connected under a sharp edge at large $p$ and $q$ values. This is an artifact of the approach of Ref. [4], which has the following drawbacks: In the description of Ref. [4], the starting point of the meanfield calculation was the Manhattan lattice. Therefore, the description of orientations near the (011) type facets is less accurate than in the present method. But it now turns out that, in addition to the facet orientations, these are the only ones that are actually found in the equilibrium shapes at low temperatures. In addition, both mean-field approximations give the same result for the free energy of the (001) facet, so no accuracy is lost there. Finally the present mean-field approximation is much more amenable to an analytical treatment, and for instance symmetries under sublattice exchange and/or arrow reversal remain much more transparent.

\section{DISCUSSION}

The model studied in this paper is expected to give a fairly good description of surfaces of ionic crystals of bcc type, such as $\mathrm{CsCl}$. We find an equilibrium shape with extended facets and mostly sharp edges for the (001) facet at low temperatures. The shape is very rich, and exhibits critical points of various natures, such as triple, tricritical, and conical points. This richness stems from the fact that, in addition to the facet edges of the (001) and (011) facets the model also has boundaries between reconstructed and unreconstructed regions. The interplay between the former and the latter produces a rich phase diagram. There exists a fair number of other models of equilibrium shapes which have been solved either in a mean-field approximation [11] or by transfer matrix methods, but we are not aware of cases where the shape is as complex as in the case presented here. Particularly striking is the likely appearance of conical points, which, as pointed out above, have a special degeneracy, i.e. a continuous set of different slopes $p$ and $q$ coexist in such points. Indicating the coordinates of the conical point with $\left(x_{P}, y_{P}, z_{P}\right)$, from Eq. (31) we have $f(p, q)=z_{P}+p x_{P}$ $+q y_{P}$, i.e., the coexisting set of orientations $p$ and $q$ is such that their surface free energy has the form of a planar facet in the $f(p, q$,$) surface. Note that the opposite situation is much$ more common and easier to understand physically, i.e., a singular point of conical type in $f(p, q)$ produces a facet in $z(x, y)$.

Finally, it is interesting to point out that shapes of $\mathrm{NaCl}$ crystals in thermal equilibrium were investigated by Heyraud and Métois [12]. They found that the shape of the crystal is a perfect cube up to a temperature $T \approx 620^{\circ}$, and that the roughening of the crystal starts from the corners of the cube and extends toward the edges. Hence it seems that the presence of sharp facet boundaries, persisting at high temperature, is a common feature of crystals of ionic type. 


\section{ACKNOWLEDGMENTS}

It is a pleasure to thank D. J. Bukman, J. W. M. Frenken, G. Mazzeo, J. D. Shore, and M. Wortis for valuable comments and discussions. E.C. would like to thank M. Wortis for his kind hospitality at the Physics Department of Simon Fraser University, where part of this work was done. E.C. acknowledges financial support from INFM - PAIS 1999.

\section{APPENDIX A: THE FREE ENERGY OF A STEP ON THE (011) FACET}

Excitations on the (011) facet of the six vertex models are easy to study. For this orientation the vertex lattice is fully polarized, with all arrows pointing, say, up and to the right, as shown in Fig. 3. No closed loops of reversed arrows are possible, since all reversed arrows point down or to the left. The only possible excitations are infinitely long steps (or steps between two boundaries). The steps can follow only two directions, and cannot have self-intersection, so that the step free energy can be calculated exactly.

In order to calculate the free energy of an isolated step we introduce the $2 \times 2$ matrix $G(k)$ as the "lattice Green function" in momentum space for straight step segments. The matrix is written in the form

$$
G(k)=\left(\begin{array}{ll}
G_{\times \times}(k) & G_{\times \bullet}(k) \\
G_{\bullet \times}(k) & G_{\bullet \bullet}(k)
\end{array}\right),
$$

where $x$ and denote the two different types of lattice points, defining the two sublattices of the staggered six vertex model, as indicated in Fig. 3. In this notation $G_{\times \times}(k)$, for instance, stands for the sum of the Boltzmann weights of straight horizontal segments between two points of type $\times$ of length $n$, multiplied by $e^{i k n}$. The Boltzmann weight counts the energies of the changed vertices between the end points of the segment and, by convention, the energy of the turn at the beginning of the segment. The straight segments of steps have Boltzmann weights $e^{-2 \beta \delta}$ and $e^{2 \beta \delta}$ in lattice points of type $\bigcirc$ and $\times$, respectively. Turns have weights $e^{\beta \epsilon}$ in and $e^{\beta(\epsilon-2 \delta)}$ in $\times$. For $G_{\times \times}(k)$ this yields

$$
G_{\times \times}(k)=e^{\beta(\epsilon+2 \delta)} e^{-2 \beta \delta} \sum_{n=1}^{\infty} e^{2 i k n}=\frac{e^{\beta \epsilon}}{e^{-2 i k}-1} .
$$

The matrix [Eq. (A1)] is given by

$$
G(k)=\frac{e^{\beta \epsilon}}{e^{-2 i k}-1}\left(\begin{array}{cc}
1 & e^{-i k} \\
e^{-i k-2 \beta \delta} & e^{2 \beta \delta}
\end{array}\right) .
$$

The full lattice Green function is obtained by considering all possible alternating sequences of horizontal and vertical segments; the contributions of the latter can be accounted for by the same matrix $G$, with an argument $k_{v}$ instead of $k_{h}$, for the horizontal steps. One obtains

$$
\begin{aligned}
\hat{G}(\vec{k}) & =G\left(k_{h}\right)+G\left(k_{v}\right)+G\left(k_{h}\right) G\left(k_{v}\right)+G\left(k_{v}\right) G\left(k_{h}\right)+\cdots \\
& \left.=\left(1+G\left(k_{v}\right)\right) \frac{1}{1-G\left(k_{h}\right) G\left(k_{v}\right)}\left(1+G\left(k_{h}\right)\right)-1, \quad \text { (A4 }\right)
\end{aligned}
$$

where $h$ and $v$ denote the two orthogonal directions of the axes of the vertex lattice.

For the calculation of the partition function as a function of step orientation and step length, one transforms back to real space:

$$
Z(L, \phi)=\sum_{i j} \int_{-\pi}^{+\pi} \frac{d \vec{k}}{4 \pi^{2}} e^{-i k_{h} L_{h}} e^{-i k_{v} L_{v}} \hat{G}_{i j}(\vec{k}) .
$$

Here $L$ denotes the total length, and $L_{h}=L \cos \phi$ and $L_{v}$ $=L \sin \phi$. Note that we sum over all possible entries of $\hat{G}(\vec{k})$, which is a sum over all possible types of initial and final positions $(i, j=\{\times, \bigcirc\})$. Setting $z=e^{i k_{h}}$, one can perform an integration over $k_{h}$ exactly. It becomes an integration along the unit circle in the complex $z$ plane. For the remaining integral over $k_{v}$ one can use the saddle point approximation, and in the limit $L \rightarrow \infty$ one obtains an expression of the type

$$
Z(L, \phi) \sim e^{-L \beta f_{s}^{(011)}(\phi)}
$$

with $f_{s}^{(011)}(\phi)$ the step free energy per unit of length. For a step running under an angle $\phi=\pi / 4$ with the principal axes of the vertex lattice, the calculation is particularly simple: the step free energy is

$$
\beta f_{s}^{(011)}\left(\frac{\pi}{4}\right)=-\frac{1}{\sqrt{2}} \log \left[\left(1+e^{\beta \epsilon}\right)\left(1+e^{\beta(\epsilon+2 \delta)}\right)\right] .
$$

The reason this quantity is negative is that, for convenience, we have calculated the increase of the free energy due to the presence of a step on the (011) facet, per unit of projected area onto the (001) facet. Calculating the same quantity per unit of projected area onto the (011) orientation one finds a positive step free energy. For a step of an arbitrary orientation $\phi$, the calculation becomes somewhat more complicated. The resulting step free energy is of the form

$$
\beta f_{s}^{(011)}(\phi)=-\sqrt{\cos \phi \sin \phi}\left(1+e^{2 \beta \delta}\right) e^{\beta \epsilon} .
$$

From the single step free energy one obtains the surface free energy in the neighborhood of the (011) facet, taking into account only noninteracting steps, which gives a contribution linear in the step density [13]. One finds

$$
f(p, q) \approx \delta-\frac{\sqrt{(1-|q|)(1-|p|)}}{2 \beta}\left(1+e^{2 \beta \delta}\right) e^{\beta \epsilon},
$$

which is valid to lowest order in an expansion around the (011) facet where $q=p=1$. Comparing Eq. (A9) with Eq. (14), one concludes that the mean-field free energy reproduces the exact surface free energy in the neighborhood of the (011) facet to linear order in the step density.

\section{APPENDIX B: LIMIT $\delta \rightarrow 0$}

In the limit $\delta \rightarrow 0$, the six vertex model has been solved exactly [14], and the exact solution can be compared with the mean-field results in the limit of small $e^{\beta \epsilon}$. For small values of $q$ and $p$, the exact solution yields [15] 


$$
f(p, q)=f(p=0, q=0)+\frac{r}{4 \beta}\left[p^{2}+q^{2}\right],
$$

with $\cos r \equiv 1-e^{2 \beta \epsilon} / 2$. For small $e^{\beta \epsilon}$ one has [see Ref. [14], Eq. (8.11.7)]

$$
f(p=0, q=0)=-\frac{e^{\beta \epsilon}}{2 \beta}+O\left(e^{3 \beta \epsilon / 2}\right) .
$$

Combining Eqs. (B1) and (B2), and noting that at lowest order in $e^{\beta \epsilon}$ one has $r=e^{\beta \epsilon}$, as an expression for the exact free energy one obtains

$$
f(p, q)=-\frac{e^{\beta \epsilon}}{2 \beta}\left(1-\frac{p^{2}+q^{2}}{2},\right),
$$

which agrees with the mean-field free energy [Eq. (14)] for $\delta=0$, and to lowest order in $p$ and $q$.
[1] H. van Beijeren and I. Nolden, in Structure and Dynamics of Surfaces, edited by W. Schommers and P. von Blanckenhagen (Springer-Verlag, Berlin, 1987), Vol. 2, pp. 259-300.

[2] E. D. Williams, Surf. Sci. 299/300, 502 (1994).

[3] Note that in the case of faceting a given unstable or metastable surface orientation may decay, in general, into a combination of three surface orientations.

[4] E. Carlon, H. van Beijeren, and G. Mazzeo, Phys. Rev. E 53, 5549R (1996).

[5] H. J. F. Knops, Phys. Rev. B 20, 4670 (1979); in this reference the critical properties of Hamiltonian (1), for $\epsilon, \epsilon^{\prime}>0$, are investigated through a real space renormalization group.

[6] H. van Beijeren, Phys. Rev. Lett. 38, 993 (1977).

[7] In the temperature range $T \sim \delta / k_{B}$ we are interested in, taking the limit $J_{0} \rightarrow \infty$ is of no serious consequence. The Boltzmann weight per unit length of a double step is smaller by a factor of $\exp \left[-\beta\left(4 J_{0}+\epsilon\right)\right]\left[\right.$ with a correction of $\exp \left(\beta J_{0}\right)$ at each corner], so if only $J_{0} \geqslant|\epsilon|$, height differences larger than one lattice unit will be extremely rare indeed.

[8] G. Mazzeo, E. Carlon, and H. van Beijeren, Phys. Rev. Lett. 74, 1391 (1995); E. Carlon, G. Mazzeo, and H. van Beijeren,
Phys. Rev. B 55, 757 (1997).

[9] Sharp edges like $A-T_{\text {trp }}$ have also been predicted for vicinal orientations of $\mathrm{Au}(110)$ crystals by $\mathrm{E}$. Carlon and $\mathrm{H}$. van Beijeren, Phys. Rev. Lett. 76, 4191 (1996). These were recently observed experimentally by M. Rost, R. van Gastel, and J. W. M. Frenken, Phys. Rev. Lett. 84, 1966 (2000).

[10] J. D. Shore and D. J. Bukman, Phys. Rev. Lett. 72, 604 (1994); D. J. Bukman and J. D. Shore, J. Stat. Phys. 78, 1277 (1995).

[11] C. Rottman and M. Wortis, Phys. Rev. B 29, 328 (1984); A.-C. Shi and M. Wortis, ibid. 37, 7793 (1988).

[12] J. C. Heyraud and J. J. Métois, J. Cryst. Growth 84, 503 (1987).

[13] If one keeps either $|p|$ or $|q|$ strictly equal to unity, and moves the other variable slightly away from it, $f(p, q)$ does not change. This is understood easily, because in this case the steps are perfectly straight and do not change the free energy, as argued in Section II A.

[14] R. J. Baxter, Exactly Solved Models in Statistical Mechanics (Academic Press, London, 1982).

[15] I. Nolden, J. Stat. Phys. 67, 155 (1992); Ph.D. thesis, Utrecht, 1990. 